\title{
Quantifying live bacterial densities using non-invasive optical measurements of $E$. coli
}

Eric van der Helm ${ }^{\mathrm{a}} \&$ Stephanie M. A. Redl ${ }^{\mathrm{a}}$

${ }^{\mathrm{a} O D i t y . b i o, ~ C o p e n h a g e n, ~ D e n m a r k ~}$

\begin{abstract}
Profiling the growth of bacterial cultures over time can be a tedious and error-prone process. Here, we present the development and evaluation of the use of the ODity platform to optically measure bacterial cell densities non-invasively. The digital growth data for $E$. coli MG1655 was calibrated against colony forming units $(\mathrm{CFU} / \mathrm{mL})$ obtained by plating on solid media. Diauxic-like shifts of liquid E. coli MG1655 cultures grown at $37^{\circ} \mathrm{C}$ in $\mathrm{LB}$ media were observed at densities as low as $2.9 \times 10^{7} \pm 1.2 \mathrm{CFU} / \mathrm{mL}$. The shift occurred at a significantly higher cell density $\left(6.0 \times 10^{7} \pm 1.2 \mathrm{CFU} / \mathrm{mL}\right)$ when the bacteria were cultured at $31^{\circ} \mathrm{C}$. These shifts were only short lived, $15.2 \pm 1.5$ and $20.8 \pm 1.8 \mathrm{~min}$ at $37^{\circ} \mathrm{C}$ and $31^{\circ} \mathrm{C}$, respectively, with the previous growth rate restored thereafter. We measured minimum doubling times of $17.0 \pm 1.1$ and $24.8 \pm 0.9 \mathrm{~min}$ at $37^{\circ} \mathrm{C}$ and $31^{\circ} \mathrm{C}$, respectively. These results demonstrate that the growth and growth rate of bacterial cultures can be accurately determined non-invasively using the ODity device.
\end{abstract}

\section{Introduction}

Profiling the growth of a bacterial culture is tedious - the optical density of culture samples must be measured at regular intervals over a long period of time in order to construct each growth curve. Small-scale (e.g., 1 L) fermenters have been traditionally used to assess growth. However, their cost prohibits many labs from being equipped with a sufficient number of these devices. Various DIY-solutions have previously been developed to measure the growth of cultures, such as the turbidistat ${ }^{1}$ and morbidostat ${ }^{2}$ devices. A more comprehensive review of various methods is provided by Pilizota and Yang ${ }^{3}$. Besides the significant costs of fermenters, sampling can disturb the culture conditions. Alternative approaches that involve cultivation of microbes in microplate readers do not resemble the growth conditions of cultivation in higher volumes owing to their limitations associated with 
culture volume, temperature control, gas atmosphere, mixing, and evaporation of liquid. Therefore, we have developed and assessed the capability of the ODity platform, a noninvasive optical device (Figure 1a) designed to measure the light scattering of a growing culture and quantify cell densities in real time.
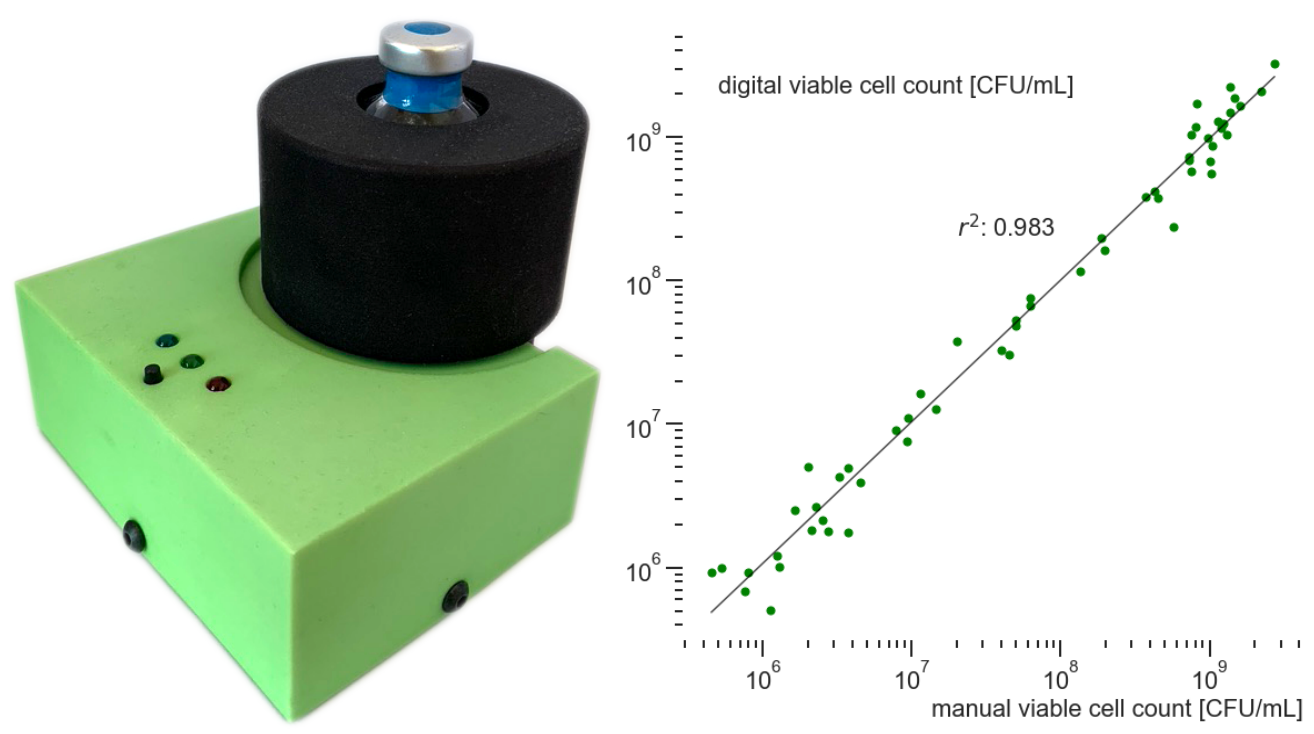

Figure 1. (a) Exterior of an ODity device D used to non-invasively measure the growth of a bacterial culture. (b) Relationship between the viable cell count as determined using counting of colony-forming units on solid media (horizontal axis) and measured using the ODity device (vertical axis). Complete linearity exists over a cell count data range across more than three orders of magnitude, with $r^{2}=0.983$ and a detection limit approaching $10^{6} \mathrm{CFU} / \mathrm{mL}$.

Although optical density (OD) is an often-used measure of cell growth, the metric has been difficult to interpret without calibration, owing to the influence of cell volume ${ }^{4}$ and cell size ${ }^{5}$ as well as its non-linear behavior 6 . Monod already noted ${ }^{7}$ this issue in 1949: "[.. in spite of the widespread use of the optical techniques, not enough efforts have been made to check them against direct estimations of cell concentrations or bacterial densities". He even goes further in highlighting the importance of using temporally sampled measurements instead of only dilutions: "Whatever instruments are used, the readings should be checked against bacterial density or cell concentration determinations, and the checks should be performed not only on different dilutions of a bacterial suspension, but at various times during the growth of a control culture. Only thus will the effects of variations of size of the cells be 
controlled. Without such controls it is impossible to decide whether the readings can be interpreted in terms of bacterial density or cell concentration, or both, or neither."

Accordingly, we measured the light scattering of a growing E. coli MG1655 culture using the ODity device and enumerated the viable cells manually by plating them onto solid media. Viable cell counts are, in contrast to estimates based on optical density, independent of the aforementioned issues of cell size and non-linear behavior. We then used the ODity calibration software to establish a linear relationship between the obtained light scattering values against viable cells (colony-forming units, $\mathrm{CFU}$ ) obtained from plating samples on solid medium (Figure 1b).

\section{Results and discussion}

E. coli growth curves

An E. coli MG1655 culture was inoculated into LB broth, starting at $10^{3}$ and $10^{4} \mathrm{CFU} / \mathrm{mL}$ and incubated at both $31^{\circ} \mathrm{C}$ and $37^{\circ} \mathrm{C}$. The viable cell counts from nine replicates are summarized in Figure 2. The lag phase is below the limit of detection and thus not shown.

\section{digital viable cell count $[\mathrm{CFU} / \mathrm{mL}]$}

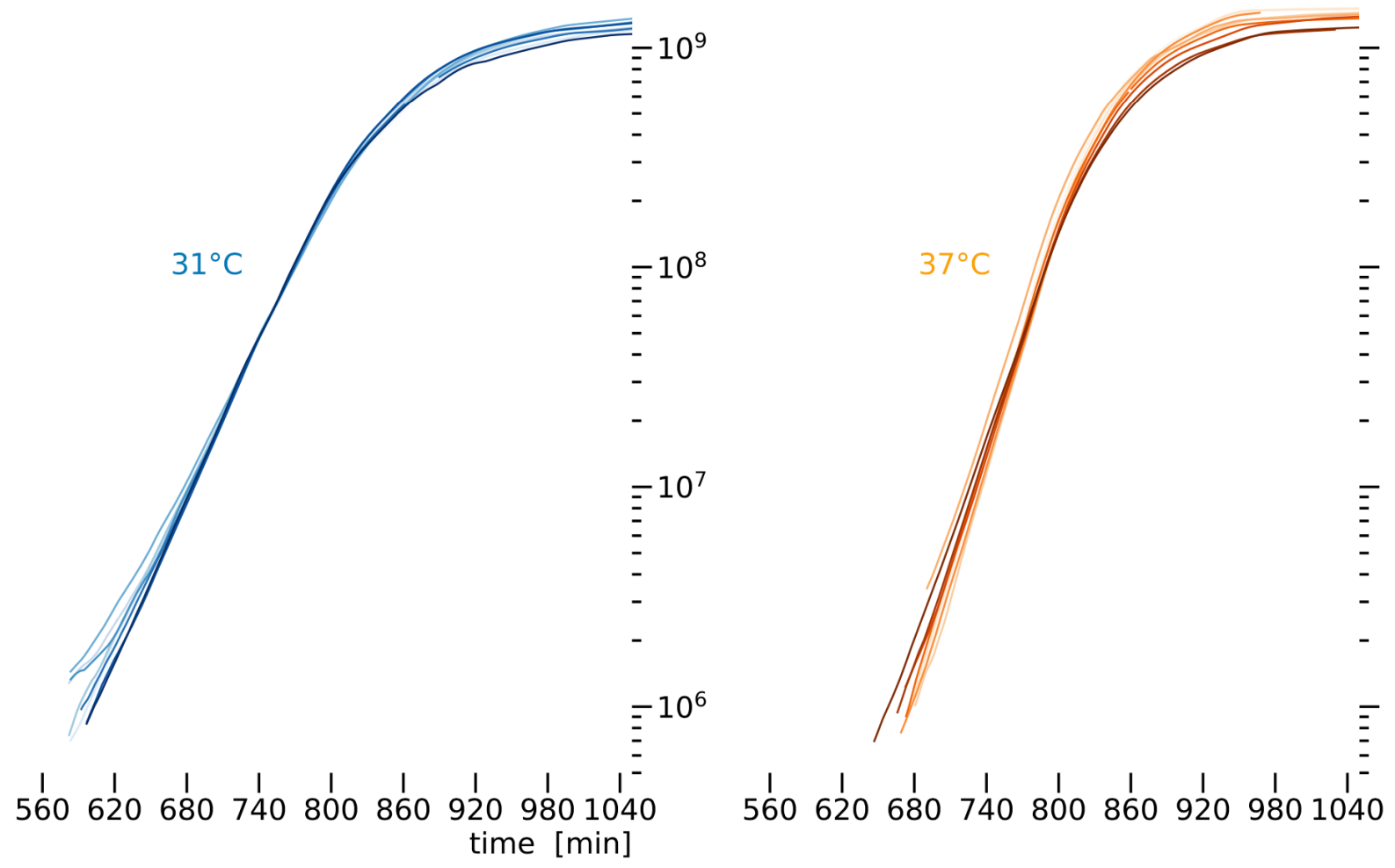

Figure 2. Examples of growth curves of E. coli MG1655 grown at $31^{\circ} \mathrm{C}$ (blue, $n=9$ ) and $37^{\circ} \mathrm{C}$ (orange, $n=9$ ) aligned in the time domain (horizontal axis). 
As measurements were recorded at 2 min intervals, the growth rate $(\mu)$ over the whole measurement could be directly calculated without fitting growth models (Figure 3 ). The growth rate $\mu$ was calculated as $\mu=\frac{\partial \ln N}{\partial t}$ (Eq. 1), where $\ln N$ is the natural logarithm of the number of cells at a given time $t$.

\section{Diauxic shifts associated with culture temperature}

In the culture grown at $37^{\circ} \mathrm{C}$, at a density of $2.9 \times 10^{7} \pm 1.2 \mathrm{CFU} / \mathrm{mL}$, a clear temporary drop in the growth rate was observed. This reduction in growth rate only lasted for $15.2 \pm 1.5$ and $20.8 \pm 1.8 \mathrm{~min}$ at $37^{\circ} \mathrm{C}$ and $31^{\circ} \mathrm{C}$, respectively (see Supplementary Figure 1), after which the previous growth rate was restored. The effect size (Hedges' $g=3.25$ ) of the duration difference between the cultures grown at $37^{\circ} \mathrm{C}$ and $31^{\circ} \mathrm{C}$ was significant $\left(p=5.2 \times 10^{-6}\right.$, Welch's $t$-test).

This diauxic phenomenon was reported first by Wang and $\mathrm{Koch}^{8}$ in 1978 and later confirmed by Sezonov ${ }^{9}$ and colleagues in 2007 . The latter researchers also offered a possible explanation: LB broth has a low concentration of sugars and thus mainly offers microbes amino acids as a carbon source. After depletion of the preferred carbon source at approximately $5 \times 10^{7} \mathrm{CFU} / \mathrm{mL}$ (corresponding to $\mathrm{OD}=0.3$, as measured by ref ${ }^{9}$ ), the cell metabolism switches to other carbon sources. However, Wang and Koch were unable to eliminate the pause in growth by supplementing LB broth with 19 L-amino acids, a trace element mixture, $\mathrm{ZnCl}_{2}$, or a mixture of adenosine, uridine, and cytidine. It should be noted that Wang and Koch utilized E. coli ML308 and not E. coli MG1655, as was used both by Sezonov and colleagues ${ }^{9}$ and in the present work.

In cultures grown at $31^{\circ} \mathrm{C}$, a similar diauxie was observed, albeit the local minimum along the growth curve was significantly ( $p=2.1 \times 10^{-4}$, Mann-Whitney $\mathrm{U}$ test) shifted upwards to 6.0 $\times 10^{7} \pm 1.2 \mathrm{CFU} / \mathrm{mL}$, compared to $2.9 \times 10^{7} \pm 1.2 \mathrm{CFU} / \mathrm{mL}$, at $37^{\circ} \mathrm{C}$. Owing to the brief and transient nature of diauxie, such diauxic shifts often go unnoticed when measurement intervals exceed $5 \mathrm{~min}$; furthermore, such shifts are not easily detected when measuring viable cells using plating (Supplementary Figure 2) and can be obscured by variation in standard laboratory procedures. 
Although LB broth is easy to prepare, it is not a defined medium, and the composition can vary even between batches. Combined with the observations of diauxic growth relatively early in the growth trajectory, this inherent variability cautions against the use of LB broth when aiming to generate quantitative or physiological measurements reproducibly.

Another interesting observation from Figure 3 is that the deceleration phase starts at the highest growth rate measured (a doubling time of $15 \mathrm{~min}$ ). This could be owing to the previously mentioned diauxie, which acts as a temporary growth-synchronization event ${ }^{10}$, however, more work is needed to elucidate this.

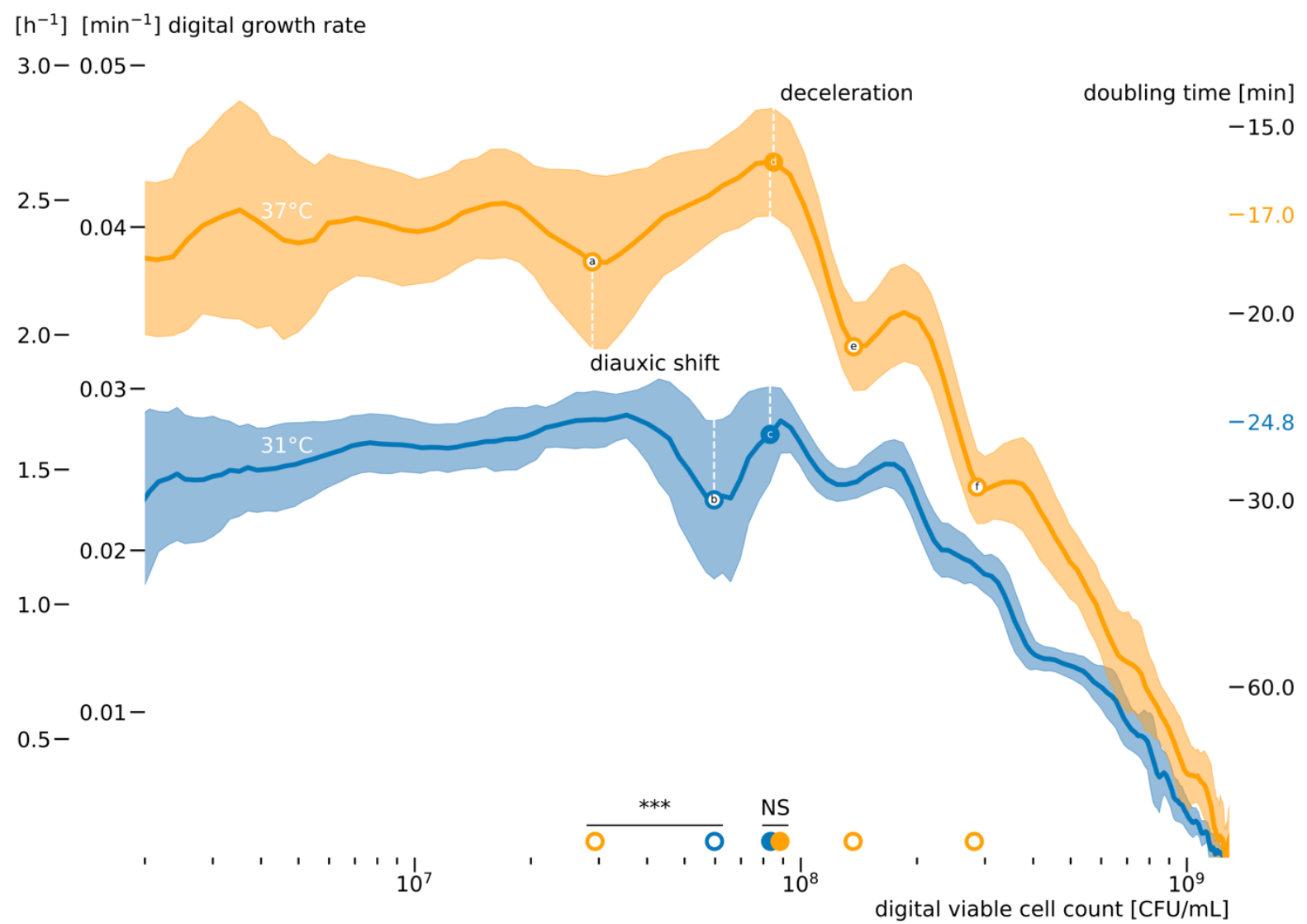

Figure 3. The digital growth rate of E. coli MG1655 cultured in LB broth at $37^{\circ} \mathrm{C}$ (orange) and $31^{\circ} \mathrm{C}$ (blue) as a function of the digital viable cell count measured using the ODity device. Shaded bands denote the standard deviation over nine biological replicates at $31^{\circ} \mathrm{C}$ (blue) and $37^{\circ} \mathrm{C}$ (orange); the growth data from Figure 2 are used throughout. When the cell density reaches the limit of detection at $10^{6} \mathrm{CFU} / \mathrm{mL}$, the culture is already in the exponential phase. At $37^{\circ} \mathrm{C}$, a temporary slowdown in growth was visible at $2.9 \times 10^{7} \pm 1.2 \mathrm{CFU} / \mathrm{mL}$ (marked with (อ) as previously reported by Sezonov and colleagues ${ }^{9}$ and attributed to a shift in carbon source utilization. At $8.8 \times 10^{7} \pm 1.1 \mathrm{CFU} / \mathrm{mL}$, the exponential phase ended, and the deceleration phase began at $37^{\circ} \mathrm{C}$ (marked with $\odot$ ), which was not significantly different ( $p=0.11$, Mann-Whitney $\mathrm{U}$ test) from the results for the $31^{\circ} \mathrm{C}$ cultures, at $8.4 \times 10^{7} \pm 1.1$ 
$\mathrm{CFU} / \mathrm{mL}$ (marked with $\odot$ ). The deceleration phase was not uniform, and in both growth profiles, more distinct diauxic shifts can be recognized, with two more observable shifts for the $37^{\circ} \mathrm{C}$ culture at $1.4 \times 10^{8} \pm 1.1$ (marked with $\odot$ ) and $2.8 \times 10^{8} \pm 1.0(\odot) \mathrm{CFU} / \mathrm{mL}$. In cultures maintained at $31^{\circ} \mathrm{C}$, a similar diauxie was observed, albeit the minimum point was significantly ( $p=2.1 \times 10^{-4}$, Mann-Whitney U test) shifted upwards to $6.0 \times 10^{7} \pm 1.2$ $\mathrm{CFU} / \mathrm{mL}$ (marked with ๑). The minimum doubling times in the exponential phase at $37^{\circ} \mathrm{C}$ and $31^{\circ} \mathrm{C}$ were $17.0 \pm 1.1$ and $24.8 \pm 0.9 \mathrm{~min}$, respectively, as shown on the right axis. The layout of Figure 3 is analogous to that of Figure 2 published by Wang and $\mathrm{Koch}^{8}$.

\section{Congruence of estimated growth rate with published estimates}

From these experiments, the maximum growth rate was directly calculated with Eq. 2 and Eq. 3 based on nine biological replicates, providing an estimate of minimum doubling time of $17.0 \pm 1.1 \mathrm{~min}$ at $37^{\circ} \mathrm{C}$ in $\mathrm{LB}$ broth.

This agrees with previous published reports (Table 1). When using the manually obtained viable cell counts by plating and fitting a four-parameter Baranyi-Roberts growth model ${ }^{11}$ (Supplementary Figure 2), we obtain a maximum growth rate of $0.035 \pm 0.001 \mathrm{~min}^{-1}$, which is equivalent to a minimum doubling time of $19.8 \mathrm{~min}$. Additionally, at $31^{\circ} \mathrm{C}$, we obtain a maximum growth rate of $0.027 \pm 0.001 \mathrm{~min}^{-1}$, which is equivalent to a minimum doubling time of 25.4 min. The Baranyi-Roberts growth model does not account for the temporary slowdown owing to diauxie and thereby slightly overestimates the minimum doubling time (and thus underestimates the maximum growth rate).

Table 1. Comparison of minimum doubling time of E. coli MG1655 cultured in $\mathrm{LB}$ at $37^{\circ} \mathrm{C}$ from selected references

\begin{tabular}{lllll}
\hline $\begin{array}{l}\text { Minimum } \\
\text { doubling } \\
\text { time }\end{array}$ & & Reference & Method & BIOnumbers \\
\hline 17.0 & \pm 1.1 & This study & Non-invasive high temporal resolution \\
& & & measurements using ODity & \\
\hline 18.1 & \pm 0.52 & $\operatorname{Ref}^{12}$ & Time-lapse microscopy of 30 cells & 104331 \\
& & & & \\
\hline
\end{tabular}




\section{$19.8 \quad$ This study $\quad$ Baranyi-Roberts model fit of viable cell count (CFU)}

$20 \quad$ Ref $^{9} \quad$ Optical density (OD)

103514

\begin{tabular}{rlll}
\hline 22.27 & \pm 0.69 & $\operatorname{Ref}^{12}$ & Optical density (OD) \\
\hline 25.7 & $\operatorname{Ref}^{4}$ & Optical density (OD) calibrated by flow \\
& & cytometry
\end{tabular}

The minimum doubling time is defined as $t_{\text {minimum doubling time }}=\frac{\ln 2}{\mu_{\max }}(E q .2)$, where the maximum growth rate is defined as $\mu_{\max }=\max \left(\frac{\partial \ln N}{\partial t}\right)$ (Eq.3), with $\ln N$ being the natural logarithm of the number of cells at a given time $t$.

\section{Conclusion}

Here, we have used a non-invasive device to calibrate the measured light scattering of viable cells in a growing culture. As an example, we measure the growth of the model organism $E$. coli MG1655 in LB broth and found a diauxic growth minimum at $2.9 \times 10^{7} \pm 1.2$ as reported previously ${ }^{8,9}$ at $37^{\circ} \mathrm{C}$. We also showed this minimum shifts up to $6.0 \times 10^{7} \pm 1.2$ when E. coli MG1655 is grown at $31^{\circ} \mathrm{C}$. This reduction in growth rate only lasts for $15.2 \pm 1.5$ and $20.8 \pm$ $1.8 \mathrm{~min}$ at $37^{\circ} \mathrm{C}$ and $31^{\circ} \mathrm{C}$, respectively, after which the previous growth rate is restored. The short-lived nature of this shift may explain its scarce record in the published literature. The significant viable cell density at which the minimum of the diauxic shift occurs implies that the shift is temperature- and/or growth rate-dependent, and more experiments are needed to explain this phenomenon.

Furthermore, we estimated a minimum doubling time of $17.0 \pm 1.1 \mathrm{~min}$ at $37^{\circ} \mathrm{C}$ in $\mathrm{LB}$ broth during the exponential phase and found that deceleration of the exponential phase already started at $8.8 \times 10^{7} \pm 1.1 \mathrm{CFU} / \mathrm{mL}$.

Collectively, these results and their agreement with traditional methods and previously published results demonstrate that the growth and growth rate of bacterial cultures can be accurately determined non-invasively using the ODity device. 


\section{Materials and methods}

\section{Growth conditions}

The starting inoculum was prepared from a single colony of streptomycin-resistant $E$. coli MG1655 (generated by spontaneous mutation as described in ref ${ }^{13}$ ) from an LB-agar plate. The colony was diluted in sterile PBS to a final concentration of $10^{3}-10^{4} \mathrm{CFU} / \mathrm{mL}$ in $80 \mathrm{~mL}$ of sterile (pre-warmed) LB broth (with $\mathrm{NaCl}[5 \mathrm{~g} / \mathrm{L}]$, tryptone $[10 \mathrm{~g} / \mathrm{L}]$, and yeast extract [5 $\mathrm{g} / \mathrm{L}]$ in $1 \mathrm{~L}$ of sterile $\mathrm{dH}_{2} \mathrm{O}$ ) supplemented with $150 \mu \mathrm{g} / \mathrm{mL}$ streptomycin.

The cultures were grown in clear glass serum bottles that were not capped to allow for air diffusion via a permeable membrane. The bottle contained a 25-mm Teflon-coated magnetic stir bar. The serum bottle was placed in an ODity Model D platform and secured in place with a screw cap. The unit was then placed onto a IKA RCT basic S001 magnetic stir plate at $350 \mathrm{rpm}$ and moved into a semi-insulated enclosure to maintain a constant temperature.

\section{Measurements}

The culture was illuminated at $624 \mathrm{~nm}$, and scattering was measured at various angles. For the 18 experiments, a total of 6,274 measurements were recorded, separated by a median interval of 131.0 seconds.

The scattering was calibrated using the ODity cloud software against the viable cell count obtained by plating in colony forming units (CFU). Measurements were aligned in the time domain by minimizing the sum of distances of the first order derivative.

\section{Manual CFU measurements}

To enumerate the bacterial concentration, $20 \mu \mathrm{L}$ of the culture was 10 -fold serially diluted in $180 \mu \mathrm{L}$ of PBS and spotted onto LB-agar plates. Colony-forming units per milliliter of culture were calculated from the mean of two individually diluted replicates.

\section{Data processing}

Data were manipulated using Pandas ${ }^{14}, \mathrm{NumPy}^{15}$, and $\mathrm{SciPy}^{16}$. The natural logarithm was used throughout this manuscript to calculate the growth rate. The growth data was filtered using a modified Savitzky-Golay function to account for non-uniformly spaced time points. A window size of 29 datapoints before $700 \mathrm{~min}$ or 7 datapoints after $700 \mathrm{~min}$ and a second order polynomial were used to compute the output. The Savitzky-Golay filter removes noise 
while maintaining the original signal structure, resulting in a more accurate derivative (in this case growth rate).

The growth rates of the individual biological replicates in Supplementary Figure 3 were merged by creating variable width bins with nine growth rate observations per cell density. The median of the nine cell densities per bin was assigned as the bin's label. The mean and the standard deviation of the nine growth rates per bin were then used to construct Figure 3. The resulting data in Figure 3 was smoothed with a moving average (over five bins) to reduce noise. The glyphs marking the diauxic shift minima and deacceleration points were calculated as the mean from the individual biological replicates shown in Supplementary Figure 3.

The maximum growth rate was calculated as an average of the growth rate over a period between the start of the diauxic shift and 40 min prior to the start of the diauxic shift.

\section{Baranyi-Roberts growth curves}

Baranyi-Roberts growth curves were calculated as described by Baranyi and Roberts ${ }^{11}$ and, more specifically, according to their four parameter model ${ }^{17}$, in which the curvature parameters for deceleration and physiological adjustment rate are fixed, as follows:

$$
\begin{gathered}
A(t)=t+\frac{1}{v} \ln \left(\frac{e^{-v t}+q_{0}}{1+q_{0}}\right) \\
y(t)=y_{0}+\mu_{\max } A(t)-\frac{1}{m} \ln \left(1+\frac{e^{m \mu_{\max } A(t)}-1}{e^{m\left(y_{\max }-y_{0}\right)}}\right)
\end{gathered}
$$

Fixing the curvature parameters $v=\mu_{\max }$ and $m=1$ results in

$$
\begin{gathered}
A(t)=t+\frac{1}{\mu_{\max }} \ln \left(\frac{e^{-\mu_{\max } t}+q_{0}}{1+q_{0}}\right) \\
y(t)=y_{0}+\mu_{\max } A(t)-\ln \left(1+\frac{e^{\mu_{\max } A(t)}-1}{e^{y_{\max }-y_{0}}}\right)
\end{gathered}
$$

where $y_{0}$ is the initial value of abundance, $\mu_{\max }$ is the maximum growth rate (1/time), $y_{\max }$ is the carrying capacity (max. abundance), and $q_{0}$ is a parameter specifying the initial physiological state of organisms (e.g., cells).

The fitting procedure was conducting using the Python package LM-FIT v 1.0.2 ${ }^{18}$ using the Basin-hopping optimization method. The maximum growth rate or minimum doubling time reported was obtained by numerically calculating the growth rate of the resulting fit instead 
of using the $\mu_{\max }$ parameter, as an analytical solution does not exist and the $\mu_{\max }$ parameter can be inaccurate ${ }^{19}$.

\section{Statistical analysis}

The geometric mean and geometric standard deviation (SD factor) are used throughout when calculating and reporting averages of manual and digital CFU quantities.

Welch's $t$-test (as implemented in the SciPy function ttest_ind with equal_var=False) was used to compare the time duration distributions of the diauxic shift between the two different growth temperatures, as these distributions are assumed to be normally distributed but with potentially unequal variances. The effect size was calculated using Hedges' $g$, which is more suitable for smaller sample sizes than Cohen's $d$.

The minima of the diauxie for each growth rate profile were determined by searching for a local minimum in the exponential phase of the growth profile. Because the distributions are not assumed to be normal (for example, owing to the batch effect of the LB influencing the exact location of the local minima), the nonparametric Mann-Whitney $U$ test (as implemented in the SciPy function mannwhitneyu) was used to compare the growth at $37^{\circ} \mathrm{C}$ and $31^{\circ} \mathrm{C}$.

\section{Code availability}

All the code used to generate the analysis and figures is publicly available in a Python Jupyter ${ }^{20}$ notebook on GitHub (https://github.com/EvdH0/ODity-growth-E-coli) and retrievable with the DOI http://doi.org/10.5281/zenodo.4937662. For convenience, a Docker container is provided to run the Jupyter notebook without the need to install the library dependencies.

\section{Conflict of interest}

The authors are shareholders of ODity.bio IVS. 


\section{Supplementary figures}
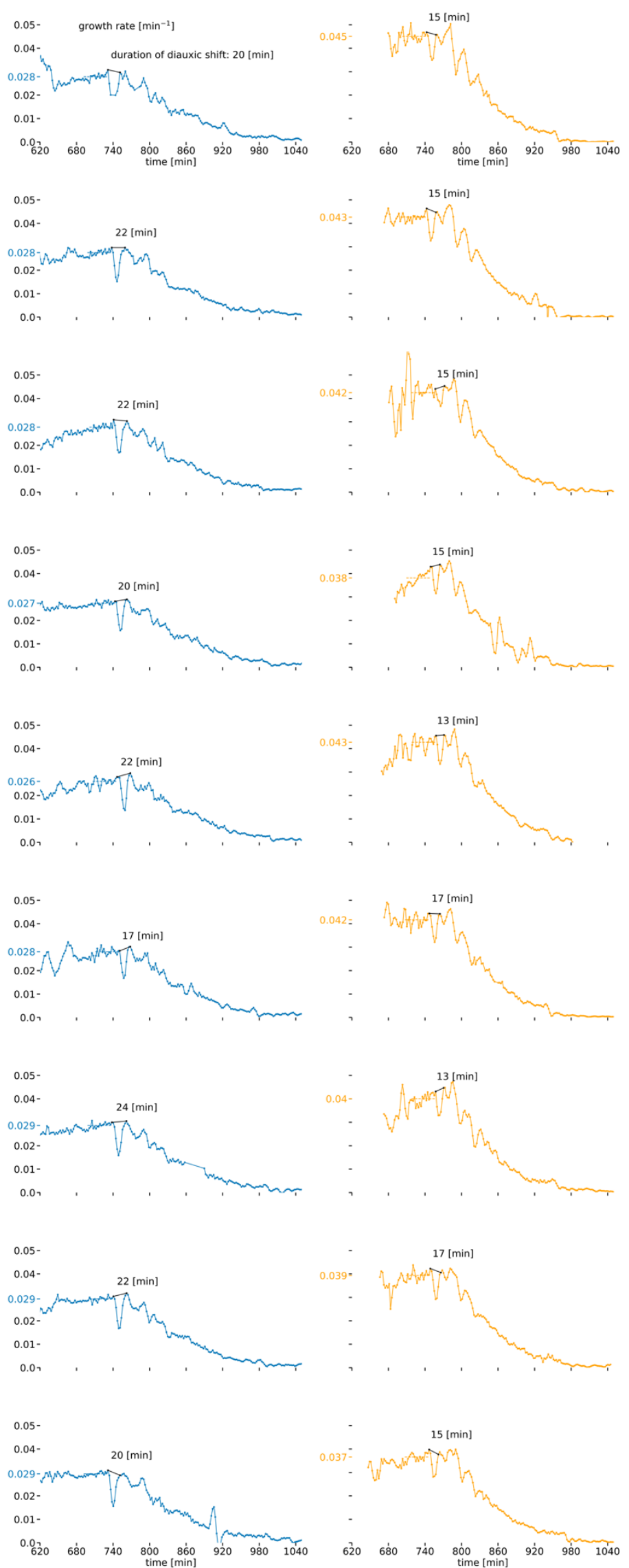
Supplementary Figure 1. Growth rate (vertical) profiles of individual experiments of E. coli MG1655 grown on LB media at $31^{\circ} \mathrm{C}$ (left) and $37^{\circ} \mathrm{C}$ (right) showing the duration of the diauxic shift (indicated by the black solid line) and the determination of the growth rate (as an average over the period covered by the dashed line). The duration of the diauxic shift is calculated by identifying the local maxima around the previously identified local minimum and finally extracting the length shown in black. The maximum growth rate (tick mark on the axis) is calculated as the average of the growth rate over a period between the start of the diauxic shift and 40 min prior to the start of the diauxic shift. This reduction in growth rate only lasts for $15.2 \pm 1.5$ and $20.8 \pm 1.8 \mathrm{~min}$ at $37^{\circ} \mathrm{C}$ and $31^{\circ} \mathrm{C}$, respectively, after which the previous growth rate is restored. The effect size (Hedges' $g=3.25$ ) of the duration difference between the cultures grown at $37^{\circ} \mathrm{C}$ and $31^{\circ} \mathrm{C}$ was significant $\left(p=5.2 \times 10^{-6}\right.$, Welch's $t$-test) 


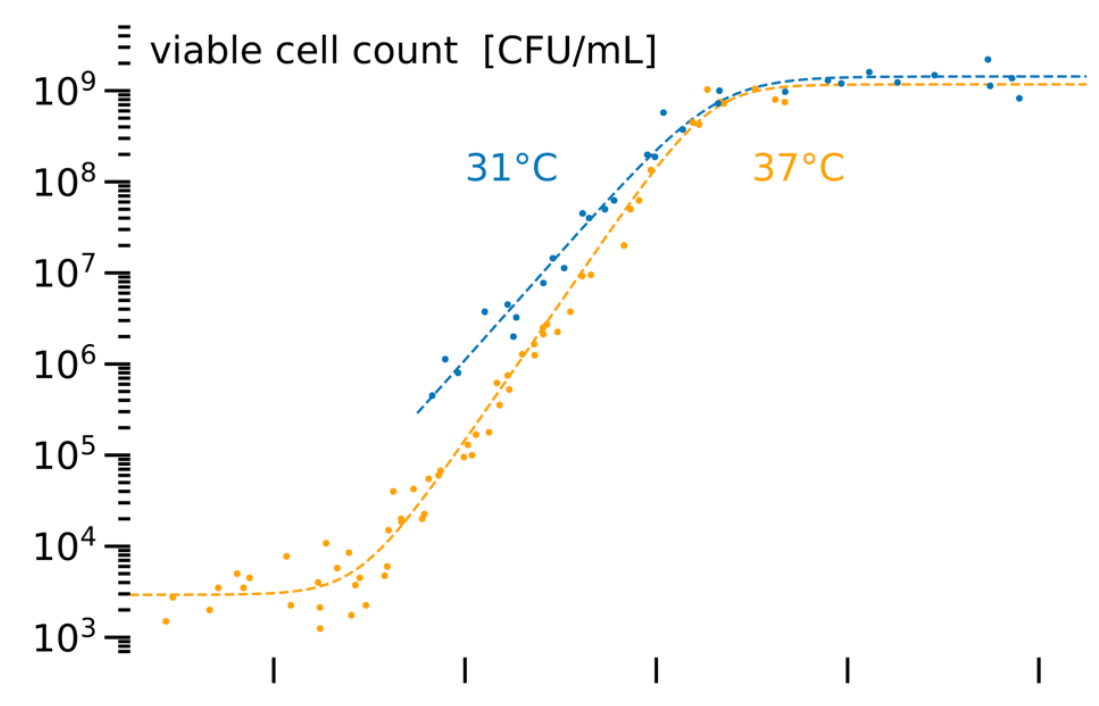

$0.04-$ growth rate $\left[\mathrm{min}^{-1}\right]$

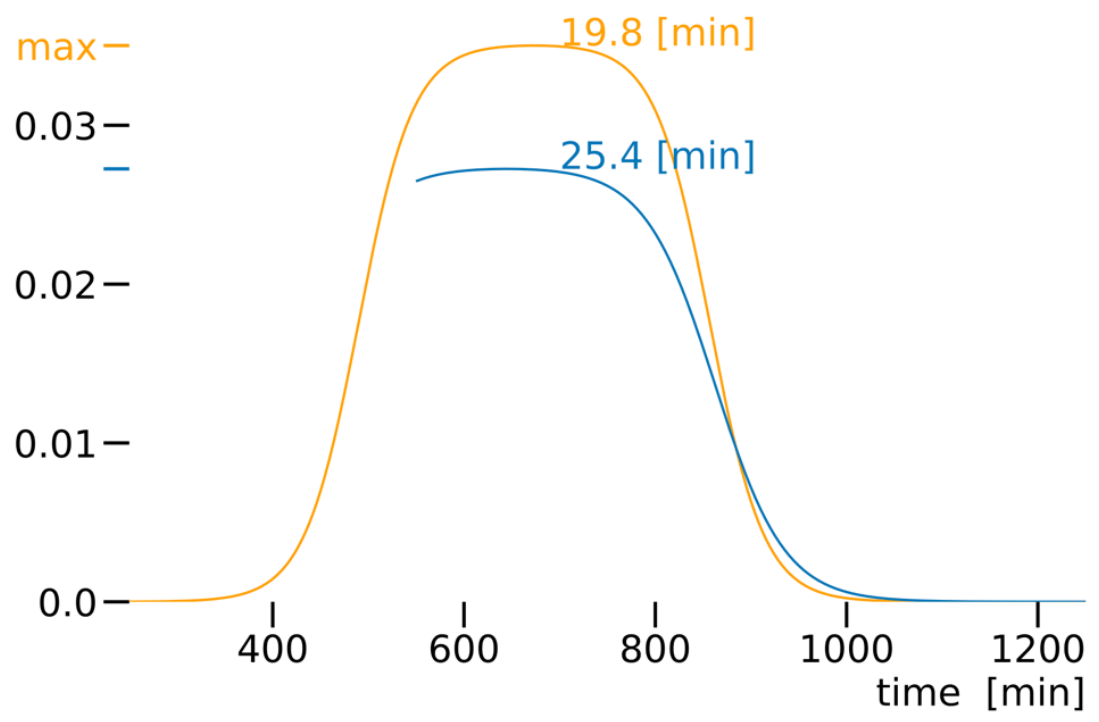

Supplementary Figure 2. Estimation of growth rate based on fitting the Baranyi-Roberts growth model. Upper panel: manually measured viable cell count $(\mathrm{CFU} / \mathrm{mL})$ based on plating of aligned growth experiments at $31^{\circ} \mathrm{C}$ (blue) and $37^{\circ} \mathrm{C}$ (orange). Growth curves are estimated using the four parameter Baranyi-Roberts model ${ }^{11,17}$, shown as dashed lines. Lower panel: numerically calculated growth rate from the Baranyi-Roberts model fit, with an estimated minimum doubling time of 19.8 and $25.4 \mathrm{~min}$ at $37^{\circ} \mathrm{C}$ and $31^{\circ} \mathrm{C}$, respectively. 


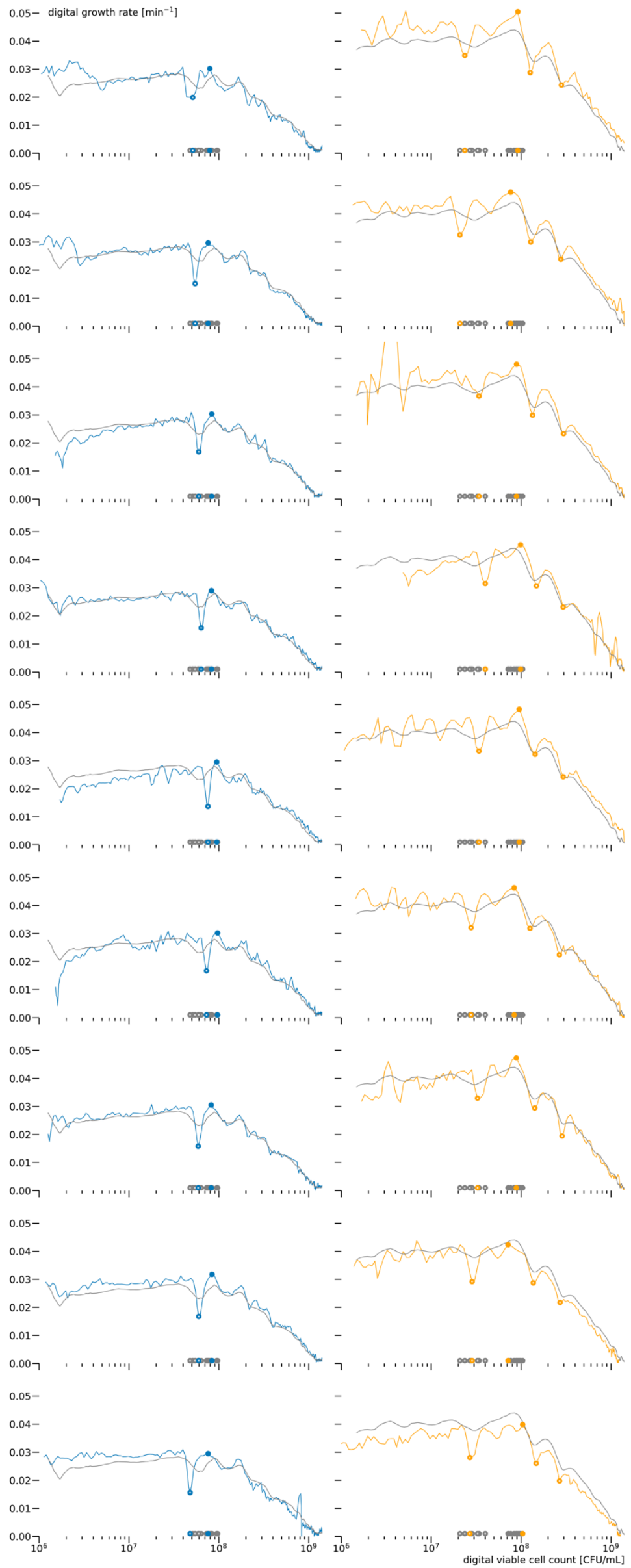


Supplementary Figure 3. Growth rate (vertical) profiles of individual experiments of $E$. coli MG1655 grown on LB media at $31^{\circ} \mathrm{C}$ (left, blue) and $37^{\circ} \mathrm{C}$ (right, orange) as a function of digital viable cell count $(\mathrm{CFU} / \mathrm{mL})$ used for the determination of the locations of the diauxic shifts and deacceleration. The local minima associated with diauxic shifts are indicated by open markers $\left(^{\circ}\right)$, and the local maxima associated with deacceleration are indicated by solid markers (.). The gray bottom markers are the result of the other biological replicates at either $31^{\circ} \mathrm{C}$ or $37^{\circ} \mathrm{C}$. The gray line denotes the average for either condition and corresponds to the average blue or orange line in Figure 3 in the main text.

\section{References}

1. Takahashi, C. N., Miller, A. W., Ekness, F., Dunham, M. J. \& Klavins, E. A Low Cost, Customizable Turbidostat for Use in Synthetic Circuit Characterization. ACS Synth. Biol. 4, 32-38 (2015).

2. Toprak, E. et al. Building a morbidostat: an automated continuous-culture device for studying bacterial drug resistance under dynamically sustained drug inhibition. Nat. Protoc. 8, 555-567 (2013).

3. Pilizota, T. \& Yang, Y.-T. "Do It Yourself” Microbial Cultivation Techniques for Synthetic and Systems Biology: Cheap, Fun, and Flexible. Front. Microbiol. 9, 1666 (2018).

4. Volkmer, B. \& Heinemann, M. Condition-Dependent Cell Volume and Concentration of Escherichia coli to Facilitate Data Conversion for Systems Biology Modeling. PLoS ONE 6, e23126 (2011)

5. Stevenson, K., McVey, A. F., Clark, I. B. N., Swain, P. S. \& Pilizota, T. General calibration of microbial growth in microplate readers. Sci. Rep. 6, 38828 (2016).

6. Begot, C., Desnier, I., Daudin, J. D., Labadie, J. C. \& Lebert, A. Recommendations for calculating growth parameters by optical density measurements. J. Microbiol. Methods 25, 225-232 (1996). 
7. Monod, J. The Growth of Bacterial Cultures. Annu. Rev. Microbiol. 3, 25 (1949).

8. Wang, C. H. \& Koch, A. L. Constancy of growth on simple and complex media. $J$. Bacteriol. 136, 969-975 (1978).

9. Sezonov, G., Joseleau-Petit, D. \& D’Ari, R. Escherichia coli Physiology in Luria-Bertani Broth. J. Bacteriol. 189, 8746-8749 (2007).

10. Boulineau, S. et al. Single-Cell Dynamics Reveals Sustained Growth during Diauxic Shifts. PLoS ONE 8, e61686 (2013).

11. Baranyi, J. \& Roberts, T. A. A dynamic approach to predicting bacterial growth in food. Int. J. Food Microbiol. 23, 277-294 (1994).

12. Reshes, G., Vanounou, S., Fishov, I. \& Feingold, M. Timing the start of division in $E$. coli : a single-cell study. Phys. Biol. 5, 046001 (2008).

13. Timms, A. R., Steingrimsdottir, H., Lehmann, A. R. \& Bridges, B. A. Mutant sequences in the rpsL gene of Escherichia coli B/r: Mechanistic implications for spontaneous and ultraviolet light mutagenesis. Mol. Gen. Genet. MGG 232, 89-96 (1992).

14. The pandas development team. pandas-dev/pandas: Pandas. (Zenodo, 2020). doi:10.5281/zenodo.3509134.

15. Harris, C. R. et al. Array programming with NumPy. Nature 585, 357-362 (2020).

16. Virtanen, P. et al. SciPy 1.0: Fundamental Algorithms for Scientific Computing in Python. Nat. Methods 17, 261-272 (2020).

17. Baranyi, J. Simple is good as long as it is enough. Food Microbiol. 14, 189-192 (1997).

18. Newville, M., Stensitzki, T., Allen, D. B. \& Ingargiola, A. LMFIT: Non-Linear LeastSquare Minimization and Curve-Fitting for Python. (Zenodo, 2014). doi:10.5281/zenodo.11813.

19. Perni, S., Andrew, P. W. \& Shama, G. Estimating the maximum growth rate from microbial growth curves: definition is everything. Food Microbiol. 22, 491-495 (2005). 
bioRxiv preprint doi: https://doi.org/10.1101/2021.06.12.448182; this version posted June 12, 2021. The copyright holder for this preprint (which was not certified by peer review) is the author/funder. All rights reserved. No reuse allowed without permission.

20. Kluyver, T. et al. Jupyter Notebooks: a publishing format for reproducible computational workflows. in Positioning and Power in Academic Publishing: Players, Agents and Agendas (eds. Loizides, F. \& Scmidt, B.) 87-90 (IOS Press, 2016). 